\title{
Publisher Correction: Process-based framework for precise neuromodulation
}

Nitzan Lubianiker, Noam Goldway (D), Tom Fruchtman-Steinbok, Christian Paret (D), Jacob N Keynan, Neomi Singer, Avihay Cohen, Kathrin Cohen Kadosh, David E J Linden and Talma Hendler (D)

Correction to: Nature Human Behaviour https://doi.org/10.1038/s41562-019-0573-y, published online 15 April 2019.

In the version of this article initially published, a typo appears in second paragraph in the section "Process-based NF specificity". The sentence "Importantly, different NF protocols (Box 1) vary in their manipulations of the general task processes." should have read "Importantly, different NF protocols (Box 2) vary in their manipulations of the general task processes." The error has been corrected in the HTML and PDF versions of the article. 